\title{
Precision Measurement of the Positron Fraction in Primary Cosmic Rays with AMS on the International Space Station
}

\author{
Valerio Vagelli* ${ }^{*}$ \\ University and INFN of Perugia, IT \\ E-mail: valerio.vagelliepg.infn.it
}

\section{Suzan Basegmez du Pree}

Institute of High Energy Physics (IHEP), Beijing, CN

\begin{abstract}
The AMS-02 experiment is a large acceptance spectrometer continuously operating onboard the International Space Station since May 2011. One of the main objectives of the AMS-02 mission is the measurement of the rare antimatter components of cosmic rays. In six years of operations, AMS-02 has detected $\sim 20$ million cosmic ray electrons and positrons that have been used to precisely measure the positron fraction - the ratio of positrons over the sum of positrons and electrons - in cosmic rays from $0.5 \mathrm{GeV}$ to $700 \mathrm{GeV}$. The analysis of the latest data collected by AMS-02 extends the energy range of the previous measurement and improves its precision.
\end{abstract}

The European Physical Society Conference on High Energy Physics

5-12 July, 2017

Venice

* Speaker.

${ }^{\dagger}$ on behalf of the AMS collaboration 
The Alpha Magnetic Spectrometer [1] (AMS-02) is the high energy spectrometer operating onboard the International Space Station since May 2011 to conduct a long duration mission of direct measurements of cosmic ray (CR) spectra in space and of their composition at the $\mathrm{TeV}$ energy scale. One of the main objectives of the AMS-02 mission is the measurement of the rare antimatter components of CRs. The accurate measurement of the relative abundance of positrons $\mathrm{e}^{+}$with respect to the total component $\left(\mathrm{e}^{+}+\mathrm{e}^{-}\right)$, the so-called positron fraction $\left(\mathrm{PF}, \mathrm{e}^{+} /\left(\mathrm{e}^{+}+\mathrm{e}^{-}\right)\right.$), provides important information on possible $\mathrm{e}^{ \pm}$exotic sources like $\mathrm{e}^{ \pm}$production from Dark Matter (DM) annihilation in the Galaxy or production from nearby astrophysical sources (pulsars and SuperNovæ Remnants). Recently, AMS-02 has released the most accurate measurement of the PF in CRs [2] based on 10.9 million $\mathrm{e}^{ \pm}$collected in the first 30 months of operations.

Differently from what expected from the production of $\mathrm{e}^{+}$in the interactions of primary CRs with the interstellar medium, the PF intensity increases above $10 \mathrm{GeV}$ providing evidence of an additional source of $\mathrm{e}^{ \pm}$in the Galaxy. For the first time, AMS-02 has observed a decrease in the spectral slope of the PF above $40 \mathrm{GeV}$, and has found a first evidence of the maximum of the PF, measured at $275 \pm 32 \mathrm{GeV}$.

AMS-02, now in orbit since more than 6 years of successful continuous operations, has collected more than $100 \times 10^{9}$ cosmic rays. In the following, we report on the status of the analysis of more than 20 million $\mathrm{e}^{ \pm}$identified in the data towards an improved updated measurement of the PF.

\section{The AMS-02 detector}

The AMS-02 detector is fully described in [1]. Its core consists of the magnetic spectrometer, composed of a permanent magnet generating a $0.14 \mathrm{~T}$ dipolar magnetic field and 9 layers of double sided silicon microstrip tracker detectors. The particle rigidity $\mathrm{R}=p / Z$, where $p$ is the momentum, is measured over a lever arm up to $3 \mathrm{~m}$. The maximum detectable rigidity for $|Z|=1$ particles is 2 TV. Four time of flight (TOF) planes trigger the readout of the detector and measure the particle flight direction and velocity. To improve the particle identification capabilities, AMS-02 is equipped from top to bottom with a transition radiation detector (TRD), a ring imaging Cherenkov detector and an electromagnetic calorimeter (ECAL). The magnet, located between TRD and ECAL, ensures that the information provided by the two subdetectors are independent from each other. The curvature measured with the tracker together with the flight direction of the particle measured with the TOF yields the sign of the charge, essential to separate antimatter from from the more abundant matter CRs.

\section{Data analysis}

Despite the direct probes to characterize the $\mathrm{e}^{+}$excess are measurements of the separate $\mathrm{e}^{-}$ and $\mathrm{e}^{+}$fluxes [3], the measurement of the PF together with the $\left(\mathrm{e}^{+}+\mathrm{e}^{-}\right)$flux [4] provides complementary information. Moreover, the separate $\mathrm{e}^{-}$and $\mathrm{e}^{+}$flux measurements have additional systematic errors due to the finite knowledge of the detector acceptance. The PF fraction measurement, instead, is limited by statistics as the acceptance uncertainties cancel out in the fraction, and together with the $\left(\mathrm{e}^{+}+\mathrm{e}^{-}\right)$flux provides a more sensitive estimation of the $\mathrm{e}^{+}$flux.

AMS-02 identifies and separates $\mathrm{e}^{ \pm}$from the dominant proton background using the electron/hadron $(\mathrm{e} / \mathrm{h})$ separation power of the TRD and of the ECAL. The signal in the 20 layers of the TRD is combined in the TRD estimator $\Lambda_{\mathrm{TRD}}=-\ln \left(\left(P_{e}\right) /\left(P_{e}+P_{p}\right)\right)$ based on the likelihood ra- 
tio for the $\mathrm{e}^{ \pm}$and proton probabilities $\left(P_{e}, P_{p}\right)$. Variables describing the topology of the shower development in the $17 \mathrm{X}_{0}$ ECAL are used as input to a BDT algorithm that evaluates the ECAL estimator $\Lambda_{\mathrm{ECAL}}$. Both $\Lambda_{\mathrm{TRD}}$ and $\Lambda_{\mathrm{ECAL}}$ have been defined and trained using pure samples of $\mathrm{e}^{-}$and protons selected from flight data using the other subdetectors of AMS-02. The distributions for $\Lambda_{\mathrm{TRD}}$ and $\Lambda_{\mathrm{ECAL}}$ and their proton rejection power are shown in Figure 1. The combined e/h rejection of AMS-02 amounts to more than $10^{6}$ in most of its energy range.

TRD
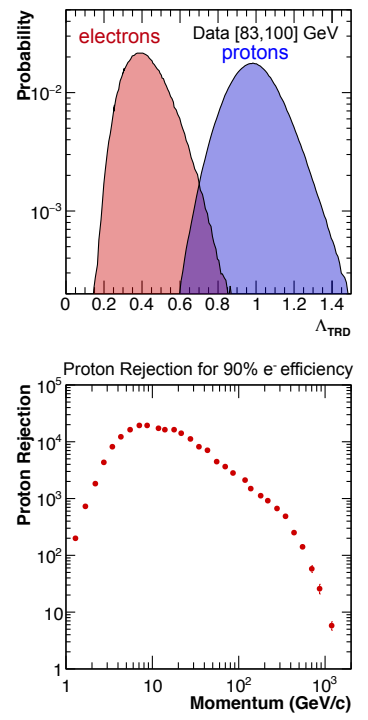

ECAL
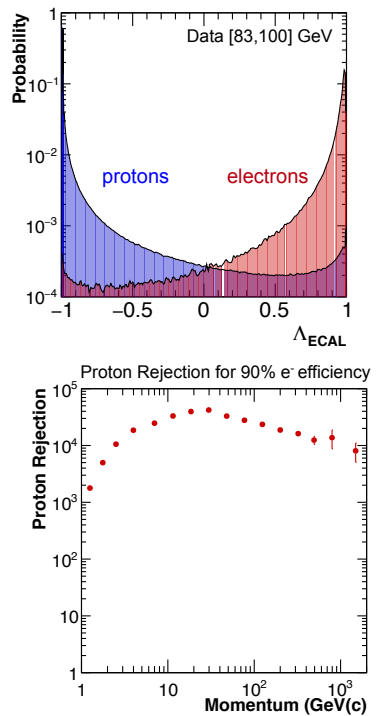

TRK
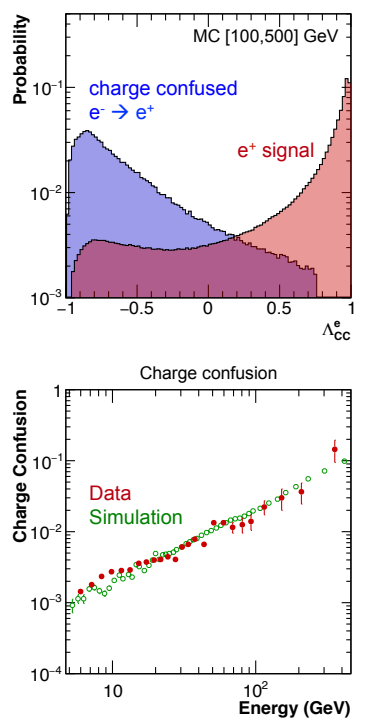

Figure 1: Left: Distribution of the TRD estimator $\Lambda_{\text {TRD }}$ for $\mathrm{e}^{ \pm}$and protons; TRD proton rejection. Center: Distribution of the ECAL estimator $\Lambda_{\mathrm{ECAL}}$ for $\mathrm{e}^{ \pm}$and protons; ECAL proton rejection; Right: Distribution of the CC estimator $\Lambda_{\mathrm{CC}}^{e}$ for signal and charge confused events; CC evaluated in flight data and in MC.

The migration of $\mathrm{e}^{-}$into the $\mathrm{e}^{+}$sample $\left(\mathrm{e}^{-} \rightarrow \mathrm{e}^{+}\right)$due to interactions with the detector materials and to the finite resolution of the tracker is evaluated using the charge confusion (CC) estimator $\Lambda_{\mathrm{CC}}$, defined as the output of a BDT algorithm trained with the MC simulation data and based on (i) the track quality (ii) noise in the detector (ii) tracker and TOF charge. The distributions for $\Lambda_{\mathrm{CC}}$ are shown in Figure 1.

The majority of protons in the data is removed using an efficient selection on $\Lambda_{\mathrm{ECAL}}$. The amount of $\mathrm{e}^{+}$and $\mathrm{e}^{-}$is then estimated fitting reference shapes in the $\Lambda_{\mathrm{CC}}: \Lambda_{\text {TRD }}$ plane for the signal $\left(\mathrm{e}^{ \pm}\right)$, the $\mathrm{CC}$ migration and the remaining protons in the positive and negative rigidity samples.

The high e/h separation capability of AMS-02 makes the uncertainty on the proton contamination negligible. The difference between the amount of $\mathrm{CC}$ confusion predicted from the MC simulation and evaluated from data, shown in Figure 1, is the major contribution to the PF systematic uncertainty in the whole energy range. Above $10 \mathrm{GeV}$, the PF measurement uncertainty is dominated by the finite $\mathrm{e}^{+}$statistics.

\section{The positron fraction measurement}

The measurement of the positron fraction by AMS-02 based on 10.9 million $\mathrm{e}^{ \pm}$collected in the first 30 months of operations is shown in Figure 2. AMS-02 has extended the accuracy of the 

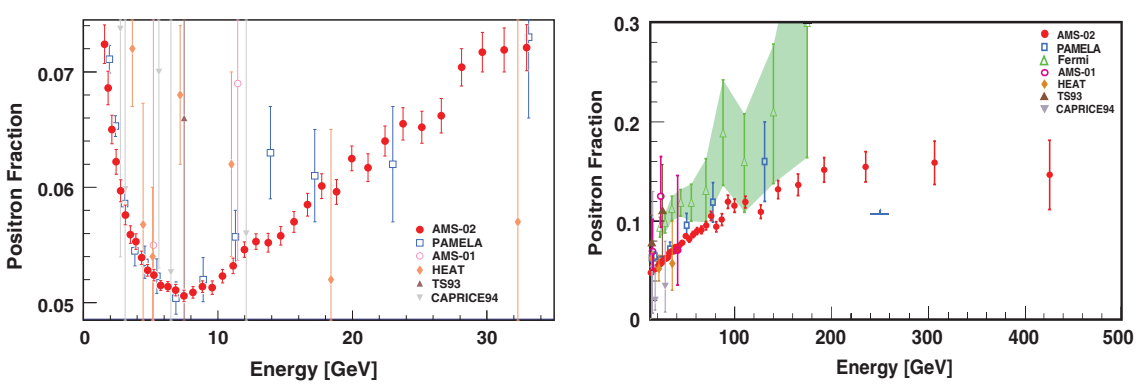

Figure 2: Positron fraction measured by AMS-02 at low and high energies with previous experiments [2].

measurement at low energy to precisely trace the rise of the PF and has extended the maximum energy reach up to $500 \mathrm{GeV}$. For the first time, AMS-02 has observed that the PF spectral slope decreases with energy, and reaches its maximum at $275 \pm 32 \mathrm{GeV}$. The accurate analysis of the PF spectral shape has provided novel information to constrain the models used to explain the origin of the $\mathrm{e}^{ \pm}$excess.

The AMS collaboration is continuously analyzing freshly collected data to extend the PF measurement. Using $\sim 20$ million CR $\mathrm{e}^{ \pm}$collected in more than 6 years of operations, the PF measurement has been extended up to $700 \mathrm{GeV}$ and its accuracy has been improved in the whole energy range. Moreover, the current statistics allow to trace the time dependence of the PF with unprecedented precision to investigate the accuracy of the astrophysical models of CR propagation and to search for charge-sign dependent effects in the solar modulation on the low energy $\mathrm{e}^{ \pm}$fluxes.

AMS-02 is currently the only magnetic spectrometer operating in space for direct detection of CRs with matter/antimatter separation capabilities, and it will continue to collect data until the end of the ISS mission. Future data will be fundamental to further extend the energy reach and the accuracy of the PF measurement. Together with other AMS-02 measurements - like the search for anisotropies in the incoming directions of $\mathrm{e}^{ \pm}$, the measurement of other rare antimatter components $(\bar{p}, \bar{D}, \ldots)$ and of composition and energy spectra of the primary and secondary CR nuclei - extended results on the PF will be fundamental to understand the processes of $\mathrm{CR}$ propagation and to further constrain, and to possibly identify, the dominating source of the $\mathrm{e}^{ \pm}$excess.

\section{Acknowledgments}

This work has been supported by acknowledged persons and institutions in AMS-02 publications ([1] and following), as well as by the Italian Space Agency under ASI-INFN Agreement No. 2013-002-R.0.

\section{References}

[1] M. Aguilar et al, Phys. Rev. Lett. 110, 141102 (2013);

[2] L. Accardo et al, Phys. Rev. Lett. 113, 121101 (2014);

[3] M. Aguilar et al, Phys. Rev. Lett. 113, 121102 (2014);

[4] M. Aguilar et al, Phys. Rev. Lett. 113, 221102 (2014). 